\title{
The metabolic syndrome and antipsychotic treatment Siegfried Kasper
}

\author{
Address: Department of Psychiatry and Psychotherapy, Medical University of Vienna, Austria \\ from International Society on Brain and Behaviour: 3rd International Congress on Brain and Behaviour \\ Thessaloniki, Greece. 28 November - 2 December 2007 \\ Published: 17 April 2008 \\ Annals of General Psychiatry 2008, 7(Suppl I):S33 doi:I0.II86/I744-859X-7-SI-S33
}

This abstract is available from: http://www.annals-general-psychiatry.com/content/7/SI/S33

(C) 2008 Kasper; licensee BioMed Central Ltd.

The metabolic syndrome is a multi-factorial disease of considerable heterogeneity but is generally considered to encompass the clustering of obesity, hypertension, impaired glucose-tolerance and dyslipidemia. It is a major cause of concern in modern medicine and it is a significant risk factor for both cardio-vascular diseases (CVD) and overall mortality and morbidity. The incidence for metabolic syndrome increases with advancing age and increasing adiposity. Patients with mental illnesses have a reduced life expectancy compared with the General Population and CVD contributes significantly to this. People with mental illnesses often have an increased risk for CVD because of a higher prevalence of obesity, smoking, diabetes and hypertension and dyslipidemia. Additionally, they have lifestyle factors that often contribute to the development of a metabolic syndrome. Patients, specifically with schizophrenia or bipolar disorders are prescribed psychotropic medications that have different weight-gain liabilities and differing risk of causing components of the metabolic syndrome. Additionally, psychotropic medications may change spontaneous activity levels due to the commonly occurring side effects of sedation and possible Parkinsonism. More scientific and clinical research is required to improve the understanding of fundamental biochemical disorders underlying the metabolical syndrome and the central nervous system and peripheral receptor biochemistry involved in the development of insulin resistance. It is apparent that psychotropic medications differ in their level of inducing metabolic syndrome and therefore the mechanisms of these psychotropic medications need to be better understood, very likely on a peripheral level of insulin resistance. 